\title{
New Classical Limits of Quantum Theories'
}

\author{
S. G. Rajeev \\ Department of Physics and Astronomy, \\ University of Rochester, \\ Rochester, New York 14627
}

\begin{abstract}
Quantum fluctuations of some systems vanish not only in the limit $\hbar \rightarrow 0$, but also as some other parameters (such as $\frac{1}{N}$, the inverse of the number of 'colors' of a Yang-Mills theory) vanish. These lead to new classical limits that are often much better approximations to the quantum theory. We describe two examples: the familiar HartreeFock-Thomas-Fermi methods of atomic physics as well as the limit of large spatial dimension. Then we present an approach of the Hecke operators on modular forms inspired by these ideas of quantum mechanics. It explains in a simple way why the spectra of these operators tend to the spectrum of random matrices for large weight for the modular forms.
\end{abstract}

\footnotetext{
${ }^{1}$ To Appear in the Proceedings of the 70th Meeting of Mathematicians and Physicists at Strassbourg, June 2002; ed. by V. Turaev and T. Wurzbacher.

${ }^{2}$ rajeev@pas.rochester.edu
} 


\section{Contents}

$\begin{array}{lll}1 & \text { Introduction } & 3\end{array}$

2 Hartree-Fock Theory of Atoms 5

2.1 The Classical Limit of the Atom . . . . . . . . . 5

2.2 The Neo-Classical Theory of the Atom $\ldots \ldots$. . . 7

2.3 Back To the Quantum Theory . . . . . . . . . . . . 11

$3 \quad$ The Thomas-Fermi Approximation 14

3.1 The Semiclassical Expansion of the Resolvent . . . . . 18

$3.2 \quad$ Derivative Expansion of Energy Function . . . . . . . 21

4 Atoms in the Limit of Large Dimension 25

4.1 The Change of Variables . . . . . . . . . . . . . . . 27

4.2 The Effective Potentia . . . . . . . . . . . . . . . . . 28

5 A Physicist's View of Modular Forms 32

5.1 The Modular Group and its Subgroups . . . . . . . . . 32

$5.2 \quad$ Modular Forms . . . . . . . . . . . . . . . . 34

5.3 Modular Forms as Wavefunctions . . . . . . . . . 37

5.4 Hecke Operators $\ldots \ldots \ldots$. . . . . . . . . . . . 41

5.5 Hecke Operators on Periodic Functions . . . . . . . . . 47

5.6 Toeplitz Operators . . . . . . . . . . . . . . . . . 49

5.7 Connection to Random Matrices . . . . . . . . . . 50

5.8 The limit of Large Weight . . . . . . . . . . . 52

5.9 The Poisson Algebra of Modular invariant functions . 54 


\section{Introduction}

It is well-known that a classical mechanical system has many possible quantizations. The classical theory is the limiting case as $\hbar \rightarrow 0$, so it is not surprising that there would be many quantum theories that in this limit reduce to the same classical theory. In this, largely expository, paper I will discuss the opposite phenomenon: how the same quantum theory can be obtained by quantizing radically different classical systems. Viewed another way, a quantum theory could depend on two parameters, say $\hbar, \lambda$ and the quantum fluctuations of some class of observables are of order $\hbar \lambda$.. Then both the limits $\hbar \rightarrow 0$ and $\lambda \rightarrow 0$ are classical theories. These classical theories could be entirely different. In an example from atomic physics, the conventional classical limit has a finite number of degrees of freedom, while the new one has an infinite number. We will refer to the the new limits (obtained by taking parameters other than $\hbar$ to zero) as 'neo-classical limits'.

This phenomenon is physically interesting because one of the new classical limits may be a better approximation to the quantum theory than the naive classical limit. These ideas came into the high energy physics literature from the work of 't Hooft and Witten on the large $\mathrm{N}$ limit of gauge theories. Witten [1] in particular worked out several simpler cases to popularize the notion that even case $N=3$ may be well approximated by the large $\mathrm{N}$ limit. But historically, the various mean field theories of condensed matter physics [2] (the spherical model for example) and even the theory of Nuclear Magnetic Resonance can be thought of as precursors of these ideas.

For example, in atomic physics, in the usual classical limit $\hbar \rightarrow 0$ 
there is no ground state: the hamiltonian is not bounded from below. However, the neo-classical limit (in this case a version of the Hartree-Fock approximation) has a ground state. Moreover, it gives an excellent first approximation to the ground state energy of the atom. The neo-semiclassical expansion gives a systematic way of calculating corrections to arbitrary accuracy, although the complexity grows rapidly with the desired accuracy.

Another important example, discussed below, is also from atomic physics: the quantum fluctuations in electron distances become small in the limit as the dimension of space becomes large. We will derive a simple effective potential that explains the stability of the atom. For another approach to this see the work of Hershbach [3].

Some important corners of mathematics are also illuminated by this phenomenon. The theory of modular forms can be viewed as the quantization of a classical mechanical system whose phase space is the upper half plane. The limit as the weight of the modular form goes to infinity corresponds to a classical limit. But there is also another classical limit corresponding to letting the level (the area of the fundamental domain ) go to infinity. These limits lead to interesting new approaches to the problem of determining the spectrum of the Hecke operators on modular forms.

The mathematical formulation of a classical dynamical system has expanded steadily in generality throughout history: as new physical theories are discovered we are led to enlarge the formalism to incorporate the new developments. In the progression from ordinary differential equations to Hamilton-Jacobi theory, symplectic geometry and the currently fashionable Poisson algebra formulation, we learn to deal 
with increasingly sophisticated systems and symmetries. Neo-classical limits produce classical systems of even greater generality, often with non-local action principles and no simple hamiltonian description [⿶]. We have described some examples of this before. There are classical limits of quantum field theories that retain asymptotically freedom and require a renormalization [5]. The challenge of finding the right mathematical description of these new kinds of classical systems remains.

Much of the story told in this paper is, of course, well-known. I hope that organizing them in this way will help to understand common themes in apparently distant subjects.

\section{Hartree-Fock Theory of Atoms}

\subsection{The Classical Limit of the Atom}

We start with a basic problem of quantum mechanics that cannot be solved exactly: an atom (or ion) with more than one electron. We usually start with the classical hamiltonian

$$
H=\sum_{i=1}^{m} \frac{p_{i}^{2}}{2 \mu}-\sum_{i=1}^{m} \frac{Z e^{2}}{\left|r_{i}\right|}+\sum_{1 \leq i<j \leq m} \frac{e^{2}}{\left|r_{i}-r_{j}\right|} .
$$

As usual, $Z$ is the atomic number of the nucleus, $e$ the charge of the electron and $\mu$ its mass. For each $1 \leq i \leq m$, the position $r_{i}$ and momentum $p_{i}$ of the electron are vectors in the Euclidean space $R^{3}$. Then we pass to the quantum theory whose hamiltonian is

$$
\hat{H}=-\hbar^{2} \sum_{i=1}^{m} \frac{\nabla_{i}^{2}}{2 \mu}-\sum_{i=1}^{m} \frac{Z e^{2}}{\left|r_{i}\right|}+\sum_{1 \leq i<j \leq m} \frac{e^{2}}{\left|r_{i}-r_{j}\right|} .
$$


This is an operator on the complex Hilbert space of anti-symmetric wavefunctions $\mathcal{F}_{m}=\Lambda^{m}(\mathcal{H})$. The anti-symmetry incorporates the Pauli exclusion principle. The space of single particle wavefunctions is $\mathcal{H}=L^{2}\left(R^{3}, C^{2}\right)$; the wavefunction of each electron takes values in $C^{2}$, since it can exist in two spin states of $^{2}$.

The limit $\hbar \rightarrow 0$ is the usual classical limit of the atom. It is wellknown that this is a spectacularly bad approximation to the quantum theory of the atom. The quantum hamiltonian is self-adjoint and bounded below and hence has a well-defined ground state. Indeed the central problem of atomic physics is the determination of this ground state wavefunction and the corresponding eigenvalue. The hamiltonian of the classical limit on the other hand, has no ground state: we can let position of an electron approach the nucleus, $r_{a} \rightarrow 0$, thus descreasing the energy down to $-\infty$.

What is missing here is the uncertainty principle: in the quantum theory it is not possible to make the position of the electron close to the nucleus without making its kinetic energy large. This might suggest that there is no way to produce a classical approximation to the atom with a stable ground state.

We will now produce a completely different classical system (with an infinite number of degrees of freedom in fact) whose quantization yields exactly the above quantum theory of the atom. Moreover, it has a ground state which is even a good approximation to the quantum ground state. What we will describe is just a reformulation of the standard Hartree-Fock approximation in atomic physics. This refor-

${ }^{3}$ We will, for simplicity, ignore relativistic and spin-dependent terms in the hamiltonian. 
mulation allows a generalization to relativistic many-fermion systems which we have described elsewhere [6].

\subsection{The Neo-Classical Theory of the Atom}

There are many standard texts that discuss the material in this section, although often without the geometric interpretation in terms of the Grassmannian. See for example [0] Let $\mathcal{H}=L^{2}\left(R^{3}, C^{2}\right)$ be the familiar complex Hilbert space. We define the Grassmannian $\operatorname{Gr}_{m}(\mathcal{H})$ to be the space of linear self-adjoint trace-class projection operators of rank $m$ :

$$
\operatorname{Gr}_{m}(\mathcal{H})=\left\{\rho: \mathcal{H} \rightarrow \mathcal{H} \mid \rho^{\dagger}=\rho ; \rho^{2}=\rho ; \operatorname{tr} \rho=m\right\}
$$

It is clear that an eigenvalue of $\rho$ is equal either to zero or to one. Corresponding to each such projection operator there is a subspace of $\mathcal{H}$ of dimension $m$ : the eigenspace of $\rho$ with eigenvalue one. Conversely, each such $m$-dimensional subspace $V$ defines an orthogonal decomposition $\mathcal{H}=V \oplus V^{\perp}$; then we can construct $\rho$ as the hermitean projection operator to $V$. Thus we can see that $\operatorname{Gr}_{m}(\mathcal{H})$ is really the set of all $m$-dimensional subspaces of $\mathcal{H}$. Thus we have an infinite dimensional (but finite rank) generalization of the usual definition of the Grassmannian [8]. $\operatorname{Gr}_{m}(\mathcal{H})$ is an infinite dimensional manifold, whose tangent space is the space of rank $m$ self-adjoint operators on $\mathcal{H}$.

A classical dynamical system is specified by (i) a manifold which will be its phase space, (ii) a symplectic form on this phase space

\footnotetext{
${ }^{4}$ If a projection operator is trace class, its trace must be an integer, the dimension of the vector space it projects.
} 
which will determine the Poisson brackets, and (iii) a real function on the phase space which is its hamiltonian.

In our theory, $\operatorname{Gr}_{m}(\mathcal{H})$ is the phase space. The symplectic form on it generalizes the standard symplectic form on finite-dimensional Grassmannians. The Poisson brackets that it implies for a pair of functions is:

$$
\{f, g\}=\operatorname{tr} \rho[d f, d g]
$$

Here $d f$ is the infinitesimal variation of $f$ which can be thought of as a linear operator on $\mathcal{H}$. If we represent the operator $\rho$ by its integral kernel $\rho_{b}^{a}(x, y)$ ( where $a=1,2$ labels spin) we can write this Poisson bracket as

$$
\left\{\rho_{b}^{a}(x, y), \rho_{d}^{c}(z, u)\right\}=\delta_{b}^{c} \delta(y, z) \rho_{d}^{a}(x, u)-\delta_{d}^{a} \delta(x, u) \rho_{b}^{c}(z, y)
$$

The last piece of information is the hamiltonian, which we postulate to be

$$
\begin{aligned}
H_{1}= & \int \frac{p^{2}}{2 \mu} \tilde{\rho}(x, p) d^{3} x \frac{d^{3} p}{(2 \pi \hbar)^{3}}-\int \frac{Z e^{2}}{|x|} \rho_{a}^{a}(x, x) d^{3} x \\
& +\frac{1}{2} \int \frac{e^{2}}{|x-y|}\left[\rho_{a}^{a}(x, x) \rho_{b}^{b}(y, y)-\rho_{b}^{a}(x, y) \rho_{a}^{b}(y, x)\right] d^{3} x d^{3} y(\cdot 6)
\end{aligned}
$$

Here, $\tilde{\rho}$ is the symbol of the operator $\rho$ :

$$
\begin{aligned}
& \tilde{\rho}_{b}^{a}(x, p)=\int \rho_{b}^{a}\left(x+\frac{u}{2}, x-\frac{u}{2}\right) e^{-\frac{i}{\hbar} p \cdot u} d^{3} u, \\
& \rho_{b}^{a}(x, y)=\int \tilde{\rho}_{b}^{a}\left(\frac{x+y}{2}, p\right) e^{\frac{i}{\hbar} p \cdot(x-y)} \frac{d^{3} p}{(2 \pi \hbar)^{3}} .
\end{aligned}
$$

Clearly, $\rho_{a}^{a}(x, x)=\int \tilde{\rho}_{a}^{a}(x, p) \frac{d^{3} p}{(2 \pi \hbar)^{3}}$.

So far it is clear that this system depends on the parameters $\mu, e, Z, m$ of the of the atom. Although the dynamical variables are 
operators, and $\hbar$ appears in the formula for the hamiltonian, it is a bona fide classical dynamical system.

We will show that a quantization of this system is exactly the quantum theory of the atom. The physical meaning of the operator $\rho$ is that it is the 'density matrix' of the electrons. Indeed $\rho_{a}^{a}(x, x)$ is the number density of the electrons at the point $x ; \operatorname{tr} \rho=\int \rho_{a}^{a}(x, x) d^{3} x=m$ is the total number of electrons. More generally, $\tilde{\rho}_{a}^{a}(x, p)$ is the density of electrons of momentum $p$ and position $x$. The Pauli exclusion principle which allows for at most one electron per single particle state, becomes the condition that this density matrix be a projection operator, so that its eigenvalues can only be zero or one. Thus this classical dynamical system realizes many of the facts we usually associate with quantum theory.

The first term represents the kinetic energy and the second term the potential energy due to the nucleus. We can combine these 'singleparticle' terms in the hamiltonian into the form

$$
\operatorname{tr} \rho K, \quad K=-\frac{\hbar^{2}}{2 \mu} \nabla^{2}-\frac{Z e^{2}}{|x|} .
$$

Now, $K$ is bounded below by $E_{1}=-\frac{1}{2} \mu \frac{Z^{2} e^{4}}{\hbar^{2}}$, as we know from the elementary theory of an ion with one electron. Since $\rho$ is positive and $\operatorname{tr} \rho=m$, we see that $\operatorname{tr} \rho K \geq m E_{1}$. ( A stricter bound can be obtained using the fact that $\rho$ is a projection. But we don't need it.) This way, the system avoids the catastrophe of the conventional classical limit. 0 .

\footnotetext{
${ }^{5}$ Strictly speaking $H_{1}$ exists only on some dense domain of $\operatorname{Gr}_{m}(\mathcal{H})$. This domain should be some class of pseudo-differential operators, and is the true phase space of the
} 
The interaction of the electrons induces two kinds of terms. The first is obvious, the Coulomb energy of a charge cloud of density $e \rho_{a}^{a}(x, x)$. The last term is not so obvious-it is the 'exchange energy'. It is needed to get back the correct quantum theory (see below). By a version of the Schwarz inequality it should be possible to see that

$$
\int\left[\rho_{a}^{a}(x, x) \rho_{b}^{b}(y, y)-\rho_{b}^{a}(x, y) \rho_{a}^{b}(y, x)\right] \frac{e^{2}}{|x-y|} d^{3} x d^{3} y \geq 0 .
$$

This expresses the physical fact that the electron-electron interaction is repulsive. Thus the total hamiltonian is bounded below by at least $m E_{1}$.

To actually find the ground state of this classical system, we must vary the hamiltonian subject to the constraints on $\rho$. Such a variation of $\rho$ is always of the form $\delta \rho=-i[\rho, u]$ for some hermitean operator $u$. The condition for an extremum is then

$$
\left[\rho, d H_{1}\right]=0
$$

Here $d H_{1}=\frac{\partial H_{1}}{\partial \rho}$ is a linear operator

$$
d H_{1}=K+\mathcal{U}+\mathcal{W} .
$$

Here, $K$ is as defined above and $\mathcal{U}$ is the multiplication by the 'mean field'

$$
\mathcal{U}_{b}^{a}(x)=\delta_{b}^{a} \int \frac{Z e^{2}}{|x-y|} \rho_{a}^{a}(y, y) d^{3} y .
$$

The 'exchange energy' contributes an operator $W$ whose integral kernel is

$$
\mathcal{W}_{b}^{a}(x, y)=-\frac{Z e^{2}}{|x-y|} \rho_{b}^{a}(x, y) .
$$

system. The correct statement is that the hamiltonian is bounded below within this domain. This is a technical project that I am unable to complete. It would be interesting to produce a functional analytic realization of the physical ideas that are described here. 
Thus we can find the extremum by solving a non-linear eigenvalue problem self-consistently. We have,

$$
\rho=\sum_{a=1}^{m} \psi_{a} \otimes \psi_{a}^{\dagger}
$$

where each of the vectors $\psi_{a} \in \mathcal{H}$ is an eigenstate of $d H_{1}$.

This is exactly the Hartree-Fock approximation to the atomic ground state. We find the wave-functions that are eigenstates of some single particle hamiltonian; the potential in this hamiltonian is selfconsistently determined by postulating that $m$ of these are occupied by electrons. Our description avoids the usual Slater determinants for the wavefunction-the hamiltonian only depends on the density matrix of the electrons and not the wavefunction itself. We have shown that this way of formulating the Hartree-Fock theory allows for generalization to systems containing an infinite number of fermions such as relativistic theories [6, 5].

Our point in this paper is that this theory can be thought of as minimizing the hamiltonian of a classical system on $\operatorname{Gr}_{m}(\mathcal{H})$. This extends to the time evolution as well: the hamiltons equations of our system are the usual equations of time-dependent Hartree-Fock theory.

\subsection{Back To the Quantum Theory}

How do we quantize a system whose phase space is a Grassmannian? It is not possible to cover the Grassmannian by a single co-ordinate system, so it is inconvenient to look for canonical variables. However, the Grassmannian is a Kähler manifold, and we can apply the ideas of geometric (or Berezin-Toeplitz) quantization [9]. In an earlier paper 
(the appendix of [6]) we used the representation theory of the unitary group to quantize this theory.

Recall the situation in the case of finite dimensional Grassmannians: let $\mathcal{V}$ be a finite dimensional vector space, and $\operatorname{Gr}_{m}(\mathcal{V})$ the set of its $m$-dimensional subspaces. $\mathrm{Gr}_{m}(\mathcal{V})$ is a compact Kähler manifold. Its canonical line bundle $\mathcal{L}$ admits a hermitean metric and a connection whose curvature is just the symplectic form. ( A line bundle that admits such a metric and connection is said to be quantizable [9].) The holomorphic sections $\operatorname{Hol}(\mathcal{L})$ of this line bundle form a finite dimensional vector space isomorphic to $\Lambda^{m}(\mathcal{V})$. This space of holomorphic sections is a subspace of the Hilbert space of square-integrable sections with a projection operator $\Pi: L^{2}(\mathcal{L}) \rightarrow \operatorname{Hol}(\mathcal{L})$. These geometric facts can be used to construct a quantization [9] of the dynamical system whose phase space is $\mathrm{Gr}_{m}(\mathcal{V})$.

From any function $f: \operatorname{Gr}_{m}(\mathcal{V}) \rightarrow R$ we will construct an operator $\hat{f}: \operatorname{Hol}(\mathcal{L}) \rightarrow \operatorname{Hol}(\mathcal{L})$ by the formula

$$
\hat{f}=\Pi f .
$$

That is, we multiply a holomorphic section by the function to get a section of $\mathcal{L}$ that may not be holomorphic; then we simply project out the holomorphic part. The operator we construct this way is selfadjoint (it is just a finite dimensional hermitean matrix in fact).

In what sense is it a quantization of the dynamical system on $\mathrm{Gr}_{m}(\mathcal{V})$ ? How will we recover the classical limit? The idea [9] is that it is merely a special case of a one-parameter family of quantum theories where $\mathcal{L}$ above is replaced by $\mathcal{L}^{N}$. As long as $N$ is a positive integer the above ideas go through: there is still a projection $\Pi_{N}: L^{2}\left(\mathcal{L}^{N}\right) \rightarrow$ 
$\operatorname{Hol}\left(\mathcal{L}^{N}\right)$ to a finite dimensional space of holomorphic sections. [1 Also in the limit $N \rightarrow \infty$ the operator algebra tends to the Poisson algebra of functions in the sense that

$$
\left\|\Pi_{N} f \Pi_{N} g-\Pi_{N}(f g)\right\|=\mathrm{O}\left(\frac{1}{N}\right),
$$

and moreover

$$
\left\|i N\left[\Pi_{N} f, \Pi_{N} g\right]-\Pi_{N}(\{f, g\})\right\|=\mathrm{O}\left(\frac{1}{N}\right) .
$$

Also the operator norm of $T_{N} f$ approaches the sup norm of the function $f$.

These ideas also extend to $\operatorname{Gr}_{m}(\mathcal{H})$ when $\mathcal{V}$ is replaced by the infinite dimensional vector space $\mathcal{H}$. The technical aspects are simpler than in [10, 11], since we need only finite rank projections. We give only a very brief outline here. Any subspace of dimension $m$ can be brought to some standard subspace whose orthogonal complement is $\mathcal{H}_{\perp}$; hence $\operatorname{Gr}_{m}(\mathcal{H})$ is a coset space] $\operatorname{Gr}_{m}(\mathcal{H})=U_{0}(\mathcal{H}) / U\left(\mathcal{H}_{\perp}\right) \times$ $U(m)$. Using the trivial representation of $U\left(\mathcal{H}_{\perp}\right)$ and the determinant representation of $U(m)$, we can construct a line bundle

$$
\mathcal{L}=\left(U_{0}(\mathcal{H}) \times C\right) / U\left(\mathcal{H}_{\perp}\right) \times U(m)
$$

The holomorphic sections of this bundle can now be constructed and shown to form $\Lambda^{m}(\mathcal{H})$. It is thus clear that $\Lambda^{m}(\mathcal{H})$ is the Hilbert space

\footnotetext{
${ }^{6}$ The space $\operatorname{Hol}\left(\mathcal{L}^{N}\right)$ carries a representation of the unitary group $U(\mathcal{V})$ given by the Young diagram of height $n$ and width $N$, generalizing the completely anti-symmetric tensor representation of the case $N=1$ above.

${ }^{7} U_{0}(\mathcal{H})$ is the group of unitary transformations that mixes the standard $m$-dimensional subspace with its orthogonal complement only by a finite rank operator. It is analogous to the 'restricted Grassmannian' of Sato [12] except that it is modelled on finite rank operators rather than compact ones.
} 
of at least one way of quantizing our system on $\mathrm{Gr}_{m}(\mathcal{H})$. Indeed, the hamiltonian of the system when worked out in this way is exactly the quantum hamiltonian of the atom we had earlier.

This quantum hamiltonian is the special case as $N=1$ of a oneparameter family of theories. For $N>1$ these describe fermions that carry a 'color' quantum number, except that only observables that are invariant under $U(N)$ are realized in the Hilbert space $\operatorname{Hol}\left(\mathcal{L}^{N}\right)$. The Hartree-Fock method thus approximates the theory for $N=1$ by the neoclassical limit as $N \rightarrow \infty$. In effect $\frac{1}{N}$ measures the size of the quantum corrections.

A relativistic generalization of this theory is described in [6]. There I developed an approach to two-dimensional QCD where the large $N$ limit was realized as a classical theory. The story above appears in the appendix to that paper. Later I found that many other problems in physics and mathematics can be thought of in a unifying way as different classical limits of the same quantum theory.

\section{The Thomas-Fermi Approximation}

The problem of minimizing the energy on the Grassmannian is still a hard problem. Further approximations are needed. It turns out that there is a way to consider the limit $\hbar \rightarrow 0$ (a kind of semi-classical approximation) which yields a simpler theory. The ideas go back to the Thomas-Fermi approximation of early atomic physics 13] and have

\footnotetext{
${ }^{8}$ It is important to take $\hbar \rightarrow 0$, after the theory has been formulated on the Grassmannian as above; if we took $\hbar \rightarrow 0$ we would get a theory without a ground state, as explained above.
} 
seen several revivals. There seems to be a connection with the density functional [14] method as well. Our point of view is based on symbol calculus and was in part inspired by the work of Lieb, Thirring [15 and others on the stability of matter. We will work out explicitly the leading terms but indicate how higher order terms can be calculated systematically if needed.

It is possible to develop the theory in a more general context than in the last section without much additional workff. We therefore consider a system of $m$ fermions with the hamiltonian

$$
H=\sum_{i=1}^{m}\left[T\left(-i \partial_{i}\right)+U\left(r_{i}\right)\right]+\sum_{i<j} G\left(r_{i}, r_{j}\right) .
$$

The configuration space of each fermion is $R^{n}$. We will allow the fermions to carry a 'spin' quantum number $\sigma=1, \cdots N_{f}$. The above hamiltonian is assumed to be independent of this quantum number. (We will usually suppress the spin index). Here, $T(p)$ is the 'dispersion relation'; i.e., the dependence of kinetic energy on momentum.T $(p)$ is usually spherically symmetric. $U(x)$ is the external potential that all the fermions are subject to and $G(x, y)=G(y, x)$ is the two body potential.

In the last section we had the following special case: the dimension of space $n=3$, the spin takes takes two values, $N_{f}=2$, the kinetic energy is $T(p)=\frac{p^{2}}{2 \mu}$ and the inter-electron potential is the Coulomb potential $G(x, y)=\frac{e^{2}}{|x-y|}$. The cases $n=1,2,3$ for the dimension of space are also of interest in other contexts.

\footnotetext{
${ }^{9}$ In fact I worked this theory in this way (in 1992) to find a relativistic generalization of the Thomas-Fermi method. It remains unpublished.
} 
We have then the Hartree-Fock energy

$$
\begin{aligned}
H_{1}(\rho)= & \operatorname{Tr}(T+U) \rho+ \\
& \frac{1}{2} \int d x d y G(x, y)[\operatorname{tr} \rho(x, x) \operatorname{tr} \rho(y, y)-\operatorname{tr} \rho(x, y) \rho(y,(22)])
\end{aligned}
$$

to be minimized over all operators satisfying $\rho^{\dagger}=\rho, \rho^{2}=\rho, \operatorname{Tr} \rho=m$. We denote by $\operatorname{tr}$ the trace over flavor while Tr includes the integral over position as well. As before, the first term represents the singleparticle kinetic energy and potential energy, the second term the direct interaction and the last term the exchange interaction.

The minimization problem above leads to the variational equations

$$
\left[\rho, d H_{1}\right]=0
$$

where the Hartree-Fock self consistent hamiltonian itself depends on $\rho$. A self-consistent solution is clearlyण $\rho=\Theta\left(E_{F}-H_{1}\right)$ where the 'Fermi energy' $E_{F}$ is determined by the condition $\operatorname{Tr} \rho=m$. It is often too hard to solve this problem, so yet another approximation is needed. We could minimize over some smaller set of operators $\rho$ thereby obtaining a variational bound that is simpler to calculate. Or we could calculate the function $H_{1}(\rho)$ in a semi-classical approximation.

The essence of the Thomas-Fermi approximation (a modern version is the density functional method [14]) is a combination of these two ideas:

1. use the variational ansatz $\rho=\Theta(-h)$ where $h=t(-i \partial)+v(x)$ is a separable hamiltonian (i.e., a function of $p$ alone plus a function of $x$

\footnotetext{
${ }^{10}$ The theta-function of a self-adjoint operator $\Theta(A)$ is defined to be the projection operator to the subspace on which $A$ is positive.
} 
alone);

2. expand the energy function

$$
H_{1}(\Theta(-t-v))
$$

semiclassically

3. minimize the leading term in this expansion $H_{T F}(t, v)$ with respect to the variational parameters $t$ and $v$. (In many treatments, however, $t$ is chosen to be the same as $T(p)$ and only $v(x)$ is varied.)

The semiclassical expansion will amount to an expansion in powers of the derivatives of $v$. The above ansatz for $h$ is motivated by the form of the Hartree-Fock hamiltonian. If the two body potential $G$ is absent, the first step is automatic, since $H_{1}$ is already in this form. Even for interacting fermions, the direct interaction is already of the separable form. The indirect energy may not be separable in general, but as long as it is a monotonic function of momentum, the projection operator $\Theta\left(E_{F}-H_{1}\right)$ will agree with that of some separable hamiltonian. Thus one expects this separable ansatz to be a good approximation.

The projection operator $\Theta(h-E)$ can be expressed in terms the resolvent operator, $\frac{1}{h-E}$, since

$$
\Theta(-x)=\int_{D} \frac{d E}{2 \pi i} \frac{1}{x-E}
$$

where $D$ is a contour that surrounds the negative real axis in a counterclockwise direction. There is a semi-classical expansion for the resolvent which can be used to derive one for the projection operator $\Theta(h-E)$. 


\subsection{The Semiclassical Expansion of the Re- solvent}

To do the semiclassical expansion, it is convenient to restate the problem in terms of (matrix-valued) functions on the phase space $R^{n} \oplus R^{n}$ rather than operators on the Hilbert space $L^{2}\left(R^{n}, C^{N_{f}}\right)$. There is a systematic theory of this procedure (symbol calculus) decribed in detail in for example, Ref. [16]. The main idea is to use Weyl ordering to set up a one-one correspondence between functions on the phase space and operators on the Hilbert space. From a function $\tilde{A}(x, p)$ we construct the operator $A$ whose kernel is

$$
A(x, y)=\int \tilde{A}\left(\frac{x+y}{2}, p\right) e^{\frac{i}{\hbar} p \cdot(x-y)}[d p] .
$$

We use the abbreviation $[d p]=\frac{d p}{(2 \pi \hbar)^{n}}$. If we apply this to simple functions such as polynomials we can check that this definition corresponds to Weyl ordering. For example, the function $x p$ becomes the operator $-i \hbar \partial x+x(-i \hbar \partial)$.

Conversely, given an operator, we define its symbol to be the function

$$
\tilde{A}(x, p)=\int A\left(x+\frac{z}{2}, x-\frac{z}{2}\right) e^{-\frac{i}{\hbar} p \cdot z} d z
$$

The idea is that $p$ is the momentum conjugate to the relative coordinate of the operator kernel. The operator multiplication can now be translated into the multiplication of symbols. The result can be expressed in closed form:

$$
\tilde{A} \circ \tilde{B}(x, p)=\left\{e^{\frac{-i \hbar}{2}\left(\frac{\partial}{\partial x^{i}} \frac{\partial}{\partial p_{i}^{\prime}}-\frac{\partial}{\partial p_{i}} \frac{\partial}{\partial x^{i^{\prime}}}\right)} \tilde{A}(x, p) \tilde{B}\left(x^{\prime}, p^{\prime}\right)\right\}_{x=x^{\prime} ; p=p^{\prime}} .
$$


The trace of operators becomes an integral in phase space

$$
\operatorname{Tr} A=\operatorname{tr} \int A(x, x) d x=\operatorname{tr} \int d x[d p] \tilde{A}(x, p) .
$$

We emphasize that the algebra of symbols under the multiplication law is exactly the same (isomorphic) to the algebra of operators on a Hilbert space;i.e., no approximation is involved in replacing an operator by its symbol.

We see that to the leading order the above muliplication law is just the pointwise multiplication of the classical theory. In the next order there is a correction proportional to the Poisson bracket. If we expand the exponential,

$$
\tilde{A} \circ \tilde{B}(x, p)=\sum_{n=0}^{\infty}\left(\frac{-i \hbar}{2}\right)^{n} \frac{1}{n !}\{\tilde{A}, \tilde{B}\}_{(n)} .
$$

Here we see a sequence of generalized Poisson brackets

$$
\{\tilde{A}, \tilde{B}\}_{(n)}=\sum_{r=0}^{n}(-1)^{r} \tilde{A}_{i_{1} \cdots i_{n-r}}^{j_{1} \cdots j_{r}} \tilde{B}_{j_{1} \cdots j_{r}}^{i_{1} \cdots i_{n-r}}
$$

where $\tilde{A}^{i}=\frac{\partial \tilde{A}}{\partial p_{i}}$ and $\tilde{A}_{i}=\frac{\partial \tilde{A}}{\partial x^{i}}$ etc. $n=1$ corresponds to the usual Poisson bracket. If $\tilde{A}$ and $\tilde{B}$ commute as matrices on spin, the odd brackets are antisymmetric and the even ones are symmetric. Otherwise, there is no particular symmetry property.

Consider now the resolvent operator of a hamiltonian $h$,

$$
r(E)=\frac{1}{h-E}
$$

We will now derive a semiclassical expansion for the symbol $\tilde{r}(E)$ of this operator. The resolvent symbol satisfies

$$
\tilde{r}(E) \circ(\tilde{h}-E)=1 .
$$


Expand $r(E)$ in power series in $\hbar$ and put into the expansion of the above equation to get,

$$
\begin{gathered}
\tilde{r}(E)=\sum_{k=0}^{\infty} \tilde{r}_{(k)}(E) \hbar^{k} \\
\sum_{n=0}^{\infty} \sum_{k=0}^{\infty}\left(\frac{-i \hbar}{2}\right)^{n} \frac{1}{n !} \hbar^{k}\left\{\tilde{r}_{(k)}(E), \tilde{h}-E\right\}_{(n)}=1 .
\end{gathered}
$$

Equating the powers of $\hbar$ on both sides of this equation, we get a set of recursion relations

$$
\begin{gathered}
\tilde{r}_{(0)}(E)=(\tilde{h}-E)^{-1}, \\
\tilde{r}_{(m)}(E)=-\sum_{n=1}^{m}\left(\frac{-i}{2}\right)^{n} \frac{1}{n !}\left\{\tilde{r}_{(n-m)}(E), \tilde{h}\right\}_{(n)}(\tilde{h}-E)^{-1} .
\end{gathered}
$$

If $\tilde{h}$ is diagonal in flavor space, the odd terms $\tilde{r}_{(2 m+1)}$ vanish. The above expansion can be used to derive the usual WKB quantization conditions as well as higher order corrections to it.

Of particular interest to us is the case where $h$ is a separable operator:

$$
\tilde{h}(x, p)=t(p)+v(x) .
$$

In this case the mixed derivatives in the generalized Poisson brackets vanish and we get

$$
\left\{\tilde{r}_{(k)}, \tilde{h}\right\}_{(n)}=\tilde{r}_{(k) i_{1} \cdots i_{n}} t^{i_{1} \cdots i_{n}}+(-1)^{n} \tilde{r}_{(k)}^{i_{1} \cdots i_{n}} v_{i_{1} \cdots i_{n}}
$$


If⿴ $t(p)=p_{i} p_{i}$ as in nonrelativistic quantum mechanics, there is only one term for $n>2$,

$$
\left\{\tilde{r}_{(k)}, \tilde{h}\right\}_{(n)}=(-1)^{n} \tilde{r}_{(k)}^{i_{1} \cdots i_{n}} v_{i_{1} \cdots i_{n}}
$$

while

$$
\left\{\tilde{r}_{(k)}, \tilde{h}\right\}_{(1)}=2 p_{i} \tilde{r}_{(k) i}-\tilde{r}_{(k)}^{i} v_{i}
$$

and

$$
\left\{\tilde{r}_{(k)}, \tilde{h}\right\}_{(2)}=2 \tilde{r}_{(k) i i}+\tilde{r}_{(k)}^{i j} v_{i j}
$$

If moreover, $v$ is diagonal in flavor space, $\tilde{r}_{(1)}=0$ and

$$
\tilde{r}_{(2)}(E)=\frac{1}{2(\tilde{h}-E)^{2}}\left[\frac{v_{i} v_{i}}{\tilde{h}-E}+2\left(p_{i} v_{i}\right)^{2}-v_{i i}\right] .
$$

\subsection{Derivative Expansion of Energy Function}

Now we can rewrite the Hartree-Fock energy in terms of the symbol $\tilde{\rho}(x, p)$ of the projection operator $\rho$.

$$
\begin{aligned}
H_{1}(\tilde{\rho})= & \operatorname{tr} \int \tilde{\rho}(x, p) T(p) d x[d p]+\operatorname{tr} \int U(x) \rho(x) d x+ \\
& \frac{1}{2} \int_{1} G(x, y) \operatorname{tr} \rho(x) \operatorname{tr} \rho(y) d x d y \\
& -\frac{1}{8} \int d x \int[d p]\left[d p^{\prime}\right] \tilde{G}\left(x, p-p^{\prime}\right) \operatorname{tr} \tilde{\rho}(x, p) \tilde{\rho}\left(x, p^{\prime}\right)
\end{aligned}
$$

Here,

$$
\rho(x)=\rho(x, x)=\int[d p] \tilde{\rho}(x, p) .
$$

\footnotetext{
${ }^{11}$ We use units here such that $2 \mu=1$, to simplify the formulas.
} 
We must minimize this subject to the constraints

$$
\tilde{\rho} \circ \tilde{\rho}(x, p)=\tilde{\rho}(x, p) ; \quad \operatorname{tr} \int \tilde{\rho}(x, p) d x[d p]=m .
$$

We reiterate that although the problem has been formulated on the classical phase space, no approximation has been made yet. All the complications are in the multiplication law of the functions (hence in the quadratic constraint on $\tilde{\rho})$.

Now we put in the separable ansatz (which satisfies the constraint automatically) and expand in powers of $\hbar$. We will have

$$
\tilde{\rho}(x, p)=\sum_{k=0}^{\infty} \tilde{\rho}_{(k)}(x, p) \hbar^{k}
$$

Using the integral representation in terms of the resolvent symbol,

$$
\tilde{\rho}=\int_{D} \frac{d E}{2 \pi i} \tilde{r}(E)
$$

we get

$$
\tilde{\rho}_{(k)}=\int_{D} \frac{d E}{2 \pi i} \tilde{r}_{(k)}(E)
$$

These terms in the expansion of $\tilde{\rho}$ are distributions on the phase space involving the delta function and its derivatives, although the $\tilde{r}_{(k)}$ are ordinary functions. In the same way, we have expansions for the number density,

$$
\begin{gathered}
\rho(x)=\sum_{k=0}^{\infty} \tilde{\rho}_{(k)}(x) \hbar^{k} \\
\tilde{\rho}_{(k)}(x)=\int_{D} \frac{d E}{2 \pi i} \int[d p] \tilde{r}_{(k)}(E, x, p)
\end{gathered}
$$

and the kinetic energy $K=\operatorname{tr} \int T(p) \tilde{\rho}(x, p) d x[d p]$,

$$
K=\sum_{k=0}^{\infty} K_{(k)} \hbar^{k}
$$


Also,

$$
\tilde{q}_{(k)}=\int_{D} \frac{d E}{2 \pi i} \operatorname{tr} \int d x[d p] \tilde{r}_{(k)}(E, x, p) T(p)
$$

The direct energy can be written in terms of the density function $\rho(x)$. The exchange integral is more complicated, being quadratic in $\tilde{\rho}$; however, in most cases it is quite small and explicit calculation in higher orders is not necessary.

It is now straightforward to calculate the Thomas-Fermi energy to lowest order in the case of nonrelativistic quantum mechanics with a potential $v$ that is diagonal in flavor space. We get upon evaluating the integrals, (it is convenient to introduce a new variable by $v(x)=$ $\left.-\phi^{2}(x)\right)$,

$$
\begin{aligned}
\rho_{(0)}(x) & =\int[d p] \Theta\left(-p^{2}+\phi(x)\right)=\omega_{n}^{\prime} \frac{\phi^{n}(x)}{n}, \\
K_{(0)} & =\operatorname{tr} \int \omega_{n}^{\prime} \frac{\phi^{n+2}(x)}{n+2} d x .
\end{aligned}
$$

Here

$$
\omega_{n}^{\prime}=\frac{\omega_{n}}{(2 \pi)^{n}}=2\left[\frac{1}{4 \pi}\right]^{\frac{n}{2}} \frac{1}{\Gamma\left(\frac{n}{2}\right)}
$$

is the area of a sphere of unit radius in momentum space. The exchange integral is,to lowest order,

$$
I_{(0)}=\frac{1}{2} \int\left[d p d p^{\prime}\right] \Theta\left(\phi^{2}(x)-p^{2}\right) \Theta\left(\phi^{2}(x)-p^{\prime 2}\right) \tilde{G}\left(p-p^{\prime}\right) .
$$

With $\tilde{G}(p)=\frac{e^{2}}{p^{2}}$ as for the Coulomb interaction, we can evaluate this more explicitly by introducing spherical polar coordinates in momentum space. We get

$$
I_{(0)}=\frac{1}{2} \alpha \frac{\omega_{n}^{\prime} \omega_{n-1}}{(2 \pi)^{n}} C_{n} \int \phi^{2 n-2}(x) d x
$$


where

$$
C_{n}=\int_{0}^{1} d y \int_{0}^{1} d y^{\prime}\left(y y^{\prime}\right)^{(n-1)} \int_{0}^{\pi} d \theta \frac{\sin ^{n-2} \theta}{y^{2}+y^{\prime 2}-2 y y^{\prime} \cos \theta} .
$$

This leads to

$$
\begin{aligned}
E_{T F}(\phi)= & \operatorname{tr} \int\left[\omega_{n}^{\prime} \frac{\phi^{n+2}}{n+2}(x)-\frac{e^{2}}{2} \frac{\omega_{n}^{\prime} \omega_{n-1}}{(2 \pi)^{n}} C_{n} \phi^{2 n-2}(x)+\omega_{n}^{\prime} U(x) \frac{\phi^{n}(x)}{n}\right] d x+ \\
& \frac{1}{2} \frac{\omega_{n}^{\prime 2}}{n^{2}} \int G(x, y) \operatorname{tr} \phi(x)^{n} \operatorname{tr} \phi^{n}(y) d x d y
\end{aligned}
$$

In our expansion the exchange term appears in the lowest order. However, in atomic physics it is as small as the terms involving derivatives of $\phi$, so it is often ignored in the lowest order treatments. Also, in many discussions, the energy is expressed as a function of the density $\rho(x)$, but one can make the change of variable from the Fermi momentum $\phi(x)$ to $\rho(x)$ easily.

Now we can vary this w.r.t. to $\phi$ to get an integral equation that determines the ground state in this approximation. Actually a more convenient variable to use is the mean field induced by this electron density: in terms of it we get a differential equation instead. If the distribution is spherically symmetric, as for an atom, this becomes a second order non-linear ordinary differential equation, the celebrated Thomas-Fermi differential equation [13].

Thus it is indeed possible to take the limit as $\hbar \rightarrow 0$ on systems such as the atom and get a sensible approximation to the ground state. However, this leads to a density function in the classical phase space and not conventional classical mechanics. Moreover, it has to be derived through an intermediary that is a bona-fide classical mechanical system but of infinite dimensions. 


\section{Atoms in the Limit of Large Dimen- sion}

As another example of a neoclassical limit, again in atomic physics, we consider the limit of large spatial dimension. This idea originates in an observation of Witten that in this limit the quantum fluctuations in the rotation invariant quantities will become small. Let $r_{a i}$ for $a=1, \cdots m$ and $i=1, \cdots n$ be the positions of $m$ electrons in an atom (or ion) of atomic number $Z$. Although the physically interesting case is $n=3$ we can, as a mathematical device, extend the system to $n$ spatial dimensions. The problem of determining the ground state becomes that of minimizing

$$
\int\left[\frac{\hbar^{2}}{2 \mu} \frac{\partial \psi^{*}}{\partial r_{a i}} \frac{\partial \psi}{\partial r_{a i}}+\left(-\sum_{a} \frac{Z e^{2}}{\left|r_{a}\right|}+\sum_{1 \leq a<b \leq m} \frac{e^{2}}{\left|r_{a}-r_{b}\right|}\right)|\psi(r)|^{2}\right] \prod_{a i} d r_{a i}(58)
$$

subject to the condition that

$$
\int|\psi(r)|^{2} \prod_{a i} d r_{a i}=1
$$

Here $\psi \in \Lambda^{m}\left(L^{2}\left(R^{n}, C^{N_{f}}\right)\right)$.

Now, the hamiltonian is invariant under the rotation group $O(n) \times$ $U\left(N_{f}\right)$. We now take the limit as $n$ and $N_{f}$ tend to infinity, and recover a classical theory. When $N_{f}$ is large, we can assume that the wavefunction is completely anti-symmetric in the 'spin' indices; the position dependent part of the wavefunction is then symmetric. Indeed we can assume that this part is rotation invariant, 127 so that it depends only the invariant quantities $q_{a b}=\frac{1}{n} r_{a i} r_{b i}$.

\footnotetext{
${ }^{12}$ In the real world, the ground state wavefunction is invariant under $O(n)$ at least for the noble gases: all the shells are filled.
} 
These inner products form a positive $m \times m$ matrix. A complete set of $O(n)$ invariants are given by the remaining bilinears $\hat{L}_{a}^{b}=$ $\frac{1}{2 n}\left[r_{a j}, \frac{\hbar}{i} \frac{\partial}{\partial r_{a j}}\right]_{+}, \hat{P}^{a b}=-\frac{\hbar^{2}}{n} \frac{\partial^{2}}{\partial r_{a i} \partial r_{b i}}$. They form a representation of the symplectic Lie algebra $S p(2 n)$ :

$$
\begin{aligned}
{\left[q_{a b}, q_{c d}\right]=0 } & =\left[\hat{P}^{a b}, \hat{P}^{c d}\right] \\
{\left[\hat{L}_{b}^{a}, \hat{L}_{d}^{c}\right] } & =\frac{i \hbar}{n}\left(\delta_{b}^{c} \hat{L}_{d}^{a}-\delta_{d}^{a} \hat{L}_{b}^{c}\right) \\
{\left[\hat{L}_{b}^{a}, \hat{P}^{c d}\right] } & =\frac{i \hbar}{n}\left(\delta_{b}^{c} \hat{P}^{a d}+\delta_{b}^{d} \hat{P}^{a c}\right) \\
{\left[\hat{L}_{b}^{a}, q_{c d}\right] } & =-\frac{i \hbar}{n}\left(\delta_{c}^{a} q_{b d}+\delta_{d}^{a} q_{b c}\right) .
\end{aligned}
$$

These commutators are proportional to $\frac{\hbar}{n}$. Hence, there are two limits where the quantum fluctuations vanish: the conventional classical limit where we let $\hbar \rightarrow 0$ keeping $n$ fixed (at the value 3 for example), or the neo-classical limit where we let $n \rightarrow \infty$ keeping $\hbar$ fixed. In this neo-classical limit, the quantum observables tend to classical ones satisfying the Poisson brackets of the sympleclassical theory.ctic Lie algebra:

$$
\begin{aligned}
\left\{q_{a b}, q_{c d}\right\}= & =\left\{P^{a b}, P^{c d}\right\} \\
\left\{L_{b}^{a}, L_{d}^{c}\right] & =\hbar\left(\delta_{b}^{c} L_{d}^{a}-\delta_{d}^{a} L_{b}^{c}\right) \\
\left\{L_{b}^{a}, P^{c d}\right\} & =\hbar\left(\delta_{b}^{c} P^{a d}+\delta_{b}^{d} P^{a c}\right) \\
\left\{L_{b}^{a}, q_{c d}\right\} & =-\hbar\left(\delta_{c}^{a} q_{b d}+\delta_{d}^{a} q_{b c}\right) .
\end{aligned}
$$

These Poisson brackets will determine the neo-classical equations of motion, once the hamiltonian is determined.

There are some subtleties in determining the hamiltonian of this neo-classical theory: there is a new term in the potential arising from the change of the measure of integration. (It is possible to interpret this as a kind of 'Fischer information' while the measure determines 
a kind of 'entropy' [17]. But we don't need this idea here.). Once the correct hamiltonian has been determined, this classical theory gives a relatively simple minimization problem for the ground state energy. Here we will consider only the static limit ( time independent solution) that determines the ground state of theory.

\subsection{The Change of Variables}

Let us return to the variational problem of determining the ground state. This will reduce to the minimization of an effective potential that depends only on $q_{a b}$. First of all, we need to determine the measure of integration $\mu(q) \prod_{c \leq d} d q_{c d}:=\mu(q) d q$ determined by the change of variables $q_{a b}=\frac{1}{n} r_{a i} r_{b i}$ on the Lebesgue measure $\prod_{a i} d r_{a i}$. This can be done by evaluating the following integral in two different ways:

$$
Z(J)=\int_{q \geq 0} e^{-q_{a b} J^{a b}} \mu(q) \prod_{c \leq d} d q_{c d}=\int e^{-\frac{1}{n} r_{a i} r_{b i} J^{a b}} \prod_{c j} d r_{c j} .
$$

( Here, $J$ is a positive matrix.) On the r.h.s. we have a standard Gaussian integral yielding

$$
\int_{q \geq 0} e^{-q_{a b} J^{a b}} \mu(q) \prod_{c \leq d} d q_{c d}=k(n, m)(\operatorname{det} J)^{-\frac{n}{2}}
$$

where $k(n, m)=(\pi n)^{-\frac{n m}{2}}$ is independent of $J$.

Thus $Z(J)$ depends on $J$ only through its determinant. It follow: ${ }^{\text {T3 }}$ that $\mu(q)$ can only depend on $q$ through $\operatorname{det} q$, prompting the

\footnotetext{
13 The space of positive matrices is a homogenous space of the general linear group, since any such matrix can be mapped to the identity by the transformation $q \mapsto S q S^{T}$ with $J$ transforming dually. The transformation law of $Z(J)$ under this transformation completely determines that of $\mu(q)$ as well.
} 
ansatz $\mu(q)=\tilde{k}[\operatorname{det} q]^{\nu}$. To determine $\nu$ we note that under the transformation $q \rightarrow S q S^{T}$, the measure of integrtion transforms as $d q \mapsto[\operatorname{det} S]^{m+1} d q$. Thus

$Z(J)=\int_{q \geq 0} e^{-\operatorname{tr} q\left(S^{T} J S\right)}[\operatorname{det} q]^{\nu}[\operatorname{det} S]^{2 \nu+m+1} d q=Z\left(S^{T} J S\right)[\operatorname{det} S]^{2 \nu+m+1}$

which determines $\nu=\frac{n-m-1}{2}$.

Thus we have

$$
\|\psi\|^{2}=\tilde{k} \int_{q \geq 0}|\psi(q)|^{2}[\operatorname{det} q]^{\frac{n-m-1}{2}} d q .
$$

It is thus tempting to define a new wavefunction absorbing the determinant pf $q$ :

$$
\chi(q)=\sqrt{ } \mu(q) \psi(q), \quad\|\psi\|^{2}=\int_{q \geq 0}|\chi(q)|^{2} d q .
$$

This $\chi(q)$ is a kind of 'radial wavefunction'.

\subsection{The Effective Potential}

Now we must express the hamiltonian in terms of this $\chi$. The only calculation we need is for the gradient of the wavefunction:

$$
\begin{aligned}
\int \frac{\hbar^{2}}{2 \mu n^{2}} \frac{\partial \psi^{*}}{\partial r_{a i}} \frac{\partial \psi}{\partial r_{a i}} \prod_{b j} d r_{b j}= & \int \frac{\hbar^{2}}{2 \mu} g^{a b c d} \mu^{-\frac{1}{2}} \frac{\partial\left(\mu^{\frac{1}{2}} \chi^{*}\right)}{\partial q^{a b}} \mu^{-\frac{1}{2}} \frac{\partial\left(\mu^{\frac{1}{2}} \chi\right)}{\partial q^{c d}} d q \\
= & \int \frac{\hbar^{2}}{2 \mu} g^{a b c d}\left[\frac{\partial \chi^{*}}{\partial q^{a b}}+\frac{n-m-1}{4} \frac{\partial \log \operatorname{det} q}{\partial q^{a b}} \chi^{*}(q)\right] \\
& {\left[\frac{\partial \chi}{\partial q^{c d}}+\frac{n-m-1}{4} \frac{\partial \log \operatorname{det} q}{\partial q^{c d}} \chi(q)\right] d q }
\end{aligned}
$$

where

$$
g^{a b c d}=n^{2} \frac{\partial q^{a b}}{\partial r_{e j}} \frac{\partial q^{c d}}{\partial r_{e j}}=\delta^{a c} q^{b d}+\delta^{a d} q^{b c}+\delta^{b c} q^{a d}+\delta^{b d} q^{a c}
$$


is an induced metric on the new configuration space. Moreover we know from elementary matrix theory that

$$
\frac{\partial \log \operatorname{det} q}{\partial q^{a b}}=q_{a b}^{-1}
$$

The terms prportional to $\chi^{*} \chi$ become a correction to the potential; the terms involving one derivative of the wavefunction combine to give a total derivative that can be dropped. Those that involve the square of the derivative of $\chi$ become a new kinetic energy term.

Thus the variational problem is now to minimize

$$
\int_{q \geq 0}\left[\frac{\hbar^{2}}{2 \mu n^{2}} g^{a b c d} \frac{\partial \chi^{*}}{\partial q^{a b}} \frac{\partial \chi}{\partial q^{c d}}+\left(V_{e f f}(q)+V(q)\right)|\chi(q)|^{2}\right] d q
$$

subject to the constraint

$$
\int_{q \geq 0}|\chi(q)|^{2} d q=1
$$

Here,

$$
V_{\text {eff }}=\frac{\hbar^{2}}{2 \mu} \frac{(n-m-1)^{2}}{4 n^{2}} \operatorname{tr} q^{-1}
$$

Also, $U(q)$ is the potential energy of the electron expressed in terms of the new variables:

$$
U(q)=-\sum_{a=1}^{m} \frac{Z \alpha}{\sqrt{ } q^{a a}}+\sum_{1 \leq a<b \leq n} \frac{\alpha}{\sqrt{ }\left[q^{a a}+q^{b b}-2 q^{a b}\right]}
$$

where $\alpha=\frac{e^{2}}{\sqrt{ } n}$.

We are now ready to take the limit as $n \rightarrow \infty$, holding $\frac{e^{2}}{\sqrt{n}}=\alpha$ ( not $e^{2}$ itself!) fixed. As expected, in the new variables, the kinetic energy of the ground state wavefunction will be of order $\frac{1}{n^{2}}$. It is very important that there is now a new term in the potential energy 
( arising from the kinetic energy of the old picture) which makes it bounded below:

$$
V(q)=\frac{\hbar^{2}}{8 \mu} \operatorname{tr} q^{-1}+U(q) .
$$

In the end the correction to the potential is quite simple!.

The ground state energy in our neoclassical approximation is the minimum this function over all positive $q$. The condition for this is an algebraic equation for $q$.

The case of a hydrogenic ion is of course simplest: when $m=1, q$ is just a positive number and there is no repulsive Coulomb interaction:

$$
V(q)=\frac{\hbar^{2}}{8 \mu} q^{-1}-\frac{Z \alpha}{\sqrt{ } q}
$$

The minimum is

$$
-2 \mu \frac{Z^{2} \alpha^{2}}{\hbar^{2}}=-\frac{2}{n} \mu \frac{Z^{2} e^{4}}{\hbar^{2}}
$$

This is to be compared with the exact answer ( for $n=3$ ) of $-\frac{1}{2} \mu \frac{Z^{2} e^{4}}{\hbar^{2}}$. Thus we get roughly the correct answer: the relative error is about $\frac{1}{n}$. It should be possible to improve on this by semi-classical methods.

More generally, it is reasonable to expect (but not guaranteed) that the minimum will respect the permutation symmetry of the problem. Then we can put the ansatz that all the diagonal elements are equal (say, $q_{a a}=\rho^{2}, \forall a$ ) and that all the off-diagonal elements are also equal, (put $q_{a b}=\rho^{2} u, \forall a \neq b$ ). Then $|u| \leq 1$ by Schwarz inequality. The potential becomes in these new variables, (it is convenient to choose a kind of atomic units during such explicit calculations, $2 \mu=\hbar=\alpha=$ 1):

$$
V(\rho, u)=\frac{1}{4 \rho^{2}} f(u)-\frac{1}{\rho} g(u)
$$


Here,

$$
g(u)=m Z-\frac{m(m-1)}{2} \frac{1}{\sqrt{ }[2(1-u)]} .
$$

Moreover,

$$
f(u)=\operatorname{tr} \tilde{q}^{-1}, \quad \tilde{q}=(1-u)+u C
$$

and $C$ is the $m \times m$ matrix all of whose matrix elements are equal to one. The spectrum of $C$ is quite simple: it has an eigenvalue equal to zero with degeneracy $m-1$ and the remaining eigenvalue is just $m$. Thus we can determine the spectra of $\tilde{q}$ and $\tilde{q}^{-1}$ and hence its trace:

$$
f(u)=\frac{m-1}{1-u}+\frac{1}{1+(m-1) u} .
$$

It is simple to minimize in $\rho$ to reduce the problem to minimizing in $u$ of $-\frac{g^{2}(u)}{f(u)}$. If we change variables yet again to

$$
v=\frac{1}{\sqrt{ }[2(1-u)]}, \quad \frac{1}{2} \leq v,
$$

our approximation of the ground state energy becomes the minimum of the rational function

$$
\tilde{V}(v)=-\left[m Z-\frac{m(m-1)}{2} v\right]^{2} \frac{2 m v^{2}-(m-1)}{2 m v^{2}\left[2(m-1) v^{2}-(m-2)\right]} .
$$

This minimum can in fact be found in closed form as an algebraic function of $m$ and $Z$. But the formula (obtained by an algebraic computation program such as Mathematica) is quite complicated. But 
this formula is fit very well ${ }^{\mathbb{4}}$ in the case of a neutral atom (i.e., $m=Z$ ) by the polynomial

$E(Z)=-\left[0.00152507+0.0871987 Z-0.920957 Z+1.83211 Z^{2}\right] \frac{2 \mu e^{4}}{n \hbar^{2}}(83)$

We have restored the original units to make comparison with other methods easier.

The point of this method is that it gives an exactly solvable and reasonably accurate picture for the ground state of the atom without having to deal with complicated nonlinear differential equations. The answers are reasonable considering the simplicity of the calculations.

\section{$5 \quad$ A Physicist's View of Modular Forms}

Next we will consider an example from mathematics: the theory of modular forms. I don't claim to have solved any deep problem in this area ( of which there are many). But perhaps the point of view described will suggest new methods.

\subsection{The Modular Group and its Subgroups}

We will give only a foretaste of the theory of modular forms. See Ref. [18] for most of the proofs and precise statements of the results. Also see Ref. [19] for relations to other areas of mathematics and physics.

The group of two by two matrices with integer entries and determinant one is called $S L_{2}(Z)$; its quotient by the center, $\Gamma(1)=$

\footnotetext{
${ }^{14}$ The fit is good to a relative error of $0.01 \%$ over the range $1 \leq Z \leq 100$
} 
$S L_{2}(Z) / Z_{2}$, is the modular group. It is conventional to denote elements of $\Gamma$ as matrices $\left(\begin{array}{ll}a & b \\ c & d\end{array}\right)$, their pre-images in $S L_{2}(Z)$.

$\Gamma(1)$ acts on the upper half of the complex plane $U$ through the fractional linear transformations

$$
z \mapsto \frac{a z+b}{c z+d}
$$

A fundamental region for this action is,

$$
D=\left\{z|| z|>1,| \operatorname{Re} z \mid<\frac{1}{2}\right\}
$$

This region is a spherical triangle with vertices at $i \infty, \pm \frac{1}{2}+\frac{\sqrt{ } 3}{2} i$. The point is that translations can be used to bring any point inside the strip $|\operatorname{Re} z|<\frac{1}{2}$; and under inversion any point is equivalent to one outside the unit circle.

The modular group is generated by $S: z \mapsto-\frac{1}{z}$ and $P=S T$ : $z \mapsto-\frac{1}{z+1}$. It is obvious that $S^{2}=1, P^{3}=1$. Indeed it can be shown that $\Gamma(1)=Z_{2} * Z_{3}$ is the free product generated by these two elements-there are no other relations among these generators.

Many interesting groups appear as subgroups of the modular group. For example, the commutator subgroup of $\Gamma(1)$ is the free group on two generators; it is a normal subgroup of index 6 . Thus the modular group is both non-abelian and infinite in an essential way: free groups are the ultimate examples of such groups.

The principal congruence subgroup $\Gamma(n)$ of level $n$ consists of all elements that are equal to the identity matrix modulo $n$. Now we

\footnotetext{
${ }^{15}$ The index $[G: H]$ of a subgroup $H$ of a group $G$ is the number of elements in the coset $G / H$; alternatively, it is the number of copies of the fundamental region of $G$ that is needed to form a fundamental region of $H$.
} 
see why the modular group is called $\Gamma(1)$; its elements are of the form $\left(\begin{array}{ll}a & b \\ c & d\end{array}\right)$ with $a=d=1 \bmod n, c=b=0 \bmod n$. Any subgroup $\Gamma$ in between, $\Gamma(n) \subset \Gamma \subset \Gamma(1)$, is called a congruence subgroup of level $n$. The congruence subgroups are all of finite index. It is possible to show by a counting argument [18] that $[\Gamma(1)$ : $\Gamma(n)]=n^{3} \prod_{p \mid n}\left[1-p^{-3}\right]$. Of particular importance $\square$ is the subgroup $\Gamma_{0}(n)=\left\{\left(\begin{array}{ll}a & b \\ c & d\end{array}\right) \in \Gamma(1), c=0 \bmod n\right\}$. The index can be shown to be[18] $\left[\Gamma(1): \Gamma_{0}(n)\right]=n \prod_{p \mid n}\left[1+p^{-1}\right]$.

\subsection{Modular Forms}

A entire modular form of integer weight $k$ associated to a subgroup $\Gamma \subseteq \Gamma(1)$ is a holomorphic function on the upper half plane (including the point at $\square i \infty)$ satisfying

$$
(c z+d)^{-k} f\left(\frac{a z+b}{c z+d}\right)=f(z), \text { for }\left(\begin{array}{ll}
a & b \\
c & d
\end{array}\right) \in \Gamma .
$$

It is called a cusp form if $f(i \infty)=0$.

If the weight is even, we can think of a modular form as a covariant tensor of order $k / 2$ ('form') on $U / \Gamma$ : the condition above is the statement that $f(z)[d z]^{\frac{k}{2}}$ is invariant under $\Gamma$.

The homolomorphic sections of the canonical line bundle on $U$ ( in this case the cotangent bundle) $\mathcal{L}$ are entire functions $f(z)$ on $U$ such that $f(z) d z$ is invariant under $\Gamma$. Thus the modular forms of weight

\footnotetext{
${ }^{16}$ It is the modular forms of weight two with respect to this subgroup that appear in the Shimura-Taniyama conjecture.

17 A function $f$ is holomorphic at $i \infty$ if it has a convergent Fourier expansion $f(z)=$ $\sum_{0}^{\infty} f_{n} e^{2 \pi i n z}$. Moreover, $f(i \infty)=f_{0}$.
} 
$k$ are simply holomorphic sections of $\mathcal{L}^{\frac{k}{2}}$. If $k$ is odd these correspond to some 'spinors' on $U / \Gamma$.

An example of a modular form $\$$ of weight $2 k$ is the Eisenstein series

$$
G_{2 k}(z)=\sum_{(m, n) \neq(0,0)} \frac{1}{(m+n z)^{2 k}} .
$$

It does not vanish at $i \infty: G_{2 k}(i \infty)=2 \zeta(2 k)$.

The most famous cusp form is

$$
\Delta(z)=(2 \pi)^{12} e^{i \pi z} \prod_{n=1}^{\infty}\left[1-e^{2 \pi i n z}\right]^{12}
$$

It is of weight 12. It is nonzero everywhere except for a simple zero at $i \infty$. It appears in Ramanujan's theory of partitions of numbers. If we expand the product of the twelveth root of $\Delta$ (which is called the Dedekind $\eta$-function) we can see that it is a generating function for partitions. The partitions of large numbers is given by the asymptotic behavior as $\operatorname{Im} z \rightarrow 0$; this is an essential singularity of the function so at first this looks hopeless. However, the modular invariance relates the value of $\Delta$ at $z$ to its value at $-\frac{1}{z}$; thus the behavior at $i \infty$ (which is trivial to determine) gives the behavior as $\operatorname{Im} z \rightarrow 0$. Hardy and Ramanujan turned this rough stone of an idea into an exquisite jewel (further polished by Rademacher), deriving an asymptotic formula for partitions of large numbers.

Any modular form is a periodic function hence can be expanded in a Fourier series. These Fourier coefficients are of great interest. An example is the Ramanujan $\tau$-function, which are the Fourier coefficients

\footnotetext{
${ }^{18}$ If we don't specify $\Gamma$, we will be speaking of the modular group itself.
} 
of the modular form above,

$$
\Delta(z)=\sum_{1}^{\infty} \tau(n) e^{2 \pi i n z} .
$$

A deep conjecture of Ramanujan (proved eventually by Deligne following ideas of Grothendieck) was that

$$
|\tau(p)| \leq 2 p^{\frac{11}{2}}
$$

In the theory of partitions, this inequality gives a bound on the error term to the Hardy-Ramanujan asymptotic formula for partitions of large numbers. These error terms seem to oscillate erratically yet a bound on their magnitude follows from the above inequality.

These erratic oscilations are related to yet another interesting phenomenon: if we define $2 p^{\frac{11}{2}} \cos \theta(p)=\tau(p)$, the angles $\theta(p)$ seem to be distributed randomly according to the circular ensemble of random matrix theory. Indeed spectra of random matrices appear in many places in the theory of modular forms and related Dirichlet series (see the recent books [19]). We will seek a clarification of this phenomenon using ideas from quantum mechanics in the theory of Hecke operators.

Let $M_{k}$ be the vector space of entire modular forms of weight $k$ and $\mathcal{S}_{k}$ that of cusp forms. It is clear that $M_{k} M_{l} \subset M_{k+l}$. For $k \geq$ 12, multiplication by $\Delta$ gives a linear map $M_{k-12} \rightarrow \mathcal{S}_{k}$. Moreover, $\operatorname{dim} \mathcal{S}_{k}=\operatorname{dim} M_{k}-1$ since there is just one condition on the Fourier coefficients of a cusp form: that the zeroth one vanishes. It is possible to reduce the determination of the dimension of $M_{k}$ to small values of $k$ using these facts; see [18] for details.

With our definition of weight, there are no entire modular forms of odd weight. For $k=0$ there is just one entire modular form, the 
constant. There are none for $k=2$. For $k=4,6,8,10$ the only entire modular forms are multiples of the Eisenstein series.

For $k \geq 12$ and even,

$$
\operatorname{dim} \mathcal{S}_{k}=\left\{\begin{array}{c}
{\left[\frac{k}{12}\right] \text { if } k \neq 2 \bmod 12} \\
{\left[\frac{k}{12}\right]-1 \text { if } k=2 \bmod 12}
\end{array}\right\} .
$$

Thus for large $k$, the dimension grows linearly with weight.A way of understanding this is that the elements of $\mathcal{S}_{k}$ are holomorphic sections

of the line bundle $\mathcal{L}^{\frac{k}{2}}$; as $k$ grows this line bundle has greater Chern character allowing for more sections: it approaches a kind of classical limit.

\subsection{Modular Forms as Wavefunctions}

To a physicist, the above theory of modular forms is very reminiscent of quantum mechanics.

We can regard the upper half plane as the phase space of some classical mechanical system. The symplectic form is the Poincarè form:

$$
\omega=\frac{d x \wedge d y}{y^{2}}, \quad z=x+i y
$$

The modular group ( or one of its finite index subgroups) can be thought of a discrete gauge group, so that points related by such a transformation represent the same classical state. A wavefunction would be a holomorphic function on the upper half plane; more precisely it would be a holomorphic section of a line bundle $\mathcal{L}^{\frac{k}{2}}$ on $U / \Gamma$. Thus $\frac{k}{2}$ is analogous to the parameter $N$ in our earlier discussion of compact Kähler manifolds. The base $U / \Gamma$ is not usually a compact manifold because of the cusps ( points at infinity and points where 
the stability group is finite). Nevertheless $U / \Gamma$ has finite area hence most of the theory ought to generalize.

What is the dynamical system whose phase space is $U / \Gamma(1)$ ?. We can imagine it as a model of quantum gravity in two dimensions. There are many such models that illustrate various aspects of gravity. Here, we think of space-time as a torus. Our model of gravity is conformally invariant (not crazy since two is the critical dimension for gravity). Thus the set of metrics modulo diffeomorphisms and conformal ( Weyl) transformations is the phase space of gravity. Using a diffeomorphism that is connected to the identity and a Weyl transformation we can bring any metric to the form $d s^{2}=\left|d \theta_{1}+z d \theta_{2}\right|^{2}$, where $0 \leq \theta_{1}, \theta_{2} \leq 2 \pi$ are standard co-ordinates on the torus. Also we can choose $\operatorname{Im} z>0$. Now if we also allow for diffeomorphisms that are not connected to the identity, which are

$$
\left(\begin{array}{l}
\theta_{1} \\
\theta_{2}
\end{array}\right) \mapsto\left(\begin{array}{ll}
a & b \\
c & d
\end{array}\right)\left(\begin{array}{l}
\theta_{1} \\
\theta_{2}
\end{array}\right), \quad\left(\begin{array}{ll}
a & b \\
c & d
\end{array}\right) \in S L_{2}(Z)
$$

then the true phase space would be $U / \Gamma(1)$ : the action of $\Gamma(1)$ on $z$ is exactly the above fractional linear transformation.

What would be the meaning of a gauge group that is only a subgroup of $\Gamma(1)$ ? We might have some additional geometric object on the torus that has to be invariant as well ( like a spin structure ) that would reduce the size of the gauge group.

What would be the hamiltonian of our theory? A closed cosmology like a torus would at first not seem to have any meaningful time evolution. An asymptotically flat space-time would have a time at infinity with respect to which we can evolve its wavefunction. In closed universes in four dimensions, the Wheeler-DeWitt equation gives a 'time 
evolution' where the conformal factor of the metric itself is a kind of time variable. But we have given this up by postulating that continuous rescalings are part of the gauge group, so that the wavefunction is invariant under them.

However we can still regard time evolution as a discrete rescaling ( 'expansion') of the universe. While rescalings connected to the identity are part of the conformal group, there are certain discrete rescalings that for example double the size of the fundamental region. There are many different ways of rescaling such a region (e.g., double just one leg of the fundamental parallelogram) which individually violate modular invariance. Only by averaging over all of them would we recover modular invariance.

What we describe above is an interpretation of the Hecke operators on modular forms:they are rescalings averaged over the modular group. Because the evolution is discrete we cannot find a generator for infinitesimal transformations. The closest we get to are the prime rescalings, which cannot be decomposed as compositions of others. They yield a family of commuting hermitean operators which together play the role of the hamiltonian. In the next section we give a more detailed description of Hecke operators.

The central problem of the Hecke theory of modular forms-determining the simultaneous eigenvectors of the Hecke operators- is just like the central problem in quantum mechanics- finding the eigenfunctions of the hamiltonian. Quantum mechanics suggests some strategies to attack this Hecke problem. The limits of large weight or large level are like neo-classical and classical limits. For example, the number of linearly independent modular forms is $\mathrm{O}(\nu k)$ in the limit of either large 
index $\nu$ or large weight $k$. The number of independent states of a quantum mechanical system with a two-dimensional phase space is of order of the area of the phase space divided by $\hbar$. The fundamental region $D$ of the modular group is not compact but still has finite area with respect to the Poincare metric:

$$
A(D)=\int_{-\frac{1}{2}}^{\frac{1}{2}} d x \int_{\sqrt{ } 1-x^{2}}^{\infty} \frac{d y}{y^{2}}=\frac{\pi}{3}
$$

The fundamental region of a subgroup of index $\nu$ is then just $\nu A(D)$. Since the number of linearly independent modular forms is $\frac{k}{6}$ for large $k$, we see that the analogue of $\hbar$ in our theory is essentially $6 A(D) / k=$ $\frac{2 \pi}{k}$. For example for the subgroup $\Gamma_{0}(p)$ which has index $\sim p$ (for prime $p$ ) there should be, according to this interpretation, $\sim p k$ linearly independent modular forms. This is indeed known in the traditional theory of modular forms.

Thus we get simpler classical analogues of the theory of modular forms in these limits; we can then hope to understand the general case by asymptotic expansions in inverse powers of $k$ or $n$. In the limit of large $n$, the subgroup

$$
\Gamma_{0}(n)=\left\{\left(\begin{array}{ll}
a & b \\
c & d
\end{array}\right) \mid c=0 \bmod n ; a d-b c=1 ; a, b, c, d \in Z\right\}
$$

becomes essentially the group of translations

$$
\left(\begin{array}{cc}
1 & c \\
0 & 1
\end{array}\right) ; c \in Z, \quad z \mapsto z+c .
$$

This is a huge simplification: the invariance group becomes more 'abelian' as $n \rightarrow \infty$. In ordinary gauge theories ( such as Yang-Mills theories) the limit as the theory becomes abelian and the limit of small quantum corrections are intimately related. ( Perturbation theory is 
essentially the same as the loop expansion.) Thus one elementary strategy to understand modular forms is to study first this easy limiting case where modular forms reduce to periodic functions on the upper half plane. We will see then that this 'perturbative' limit is also a 'semi-classical limit' of large $k$.

The theory of modular forms should be viewed as a gauge theory with a non-abelian and non-compact gauge group- the modular group. It has all the essential features of the gauge theories of physics but in a much simpler mathematical setting: the group is only countably infinite instead of being an infinite dimensional Lie group. Thus there is no need for renormalization. By studying modular forms we are studying the gauge principle in its purest form without contamination by the other complications of quantum field theories. The number theory of the last century bears witness to the claim that even this simplest of all non-abelian gauge theories is very deep: some of the deepest problems of number theory could be solved if we understood the spectrum of the Hecke operators.

In this paper we develop only the analogue of lowest order pertrurbation theory ('abelian approximation'). Our other ideas on nonabelian gauge theories ('summing planar diagrams') also should have analogues here and should lead to deep results in the future. I hope an enterprising reader will take up this challenge.

\section{$5.4 \quad$ Hecke Operators}

We now return to the exposition of the classic theory of modular forms due to Hecke. Hecke was motivated by the work of Mordell who in 
turn was trying to understand the Ramanujan conjecture on the $\tau$ function.

A lattice on the complex plane is a set of points

$$
w \sim w+r \omega_{1}+s \omega_{2}, \quad r, s \in Z
$$

the fundamental region is a parallelogram with side $z$. A linear change of basis with integer coefficients and determinant one ${ }^{\mathbb{P}} a d-b c=1$,

$$
\left(\begin{array}{l}
\omega_{1} \\
\omega_{2}
\end{array}\right) \mapsto\left(\begin{array}{ll}
a & b \\
c & d
\end{array}\right)\left(\begin{array}{l}
\omega_{1} \\
\omega_{2}
\end{array}\right)
$$

does not change the lattice. By identifying the opposite sides of this parallelogram we get a torus. By a rotating our co-ordinate system and choosing an appropriate unit of length we can choose $\omega_{2}=1$. Also, we can reflect around this axis if needed to make $\omega_{1}$ lie in the upper half plane. Thus only the ratio $z=\frac{\omega_{1}}{\omega_{2}}$ is needed to specify a lattice. A modular transformation

$$
z \mapsto \frac{a z+b}{c z+d}, \quad a d-b c=1
$$

is just the effect of a change of basis on this ratio. We call this lattice $L_{z}$.

A fractional linear transformation

$$
z \mapsto \frac{a z+b}{c z+d}
$$

with integer coefficients will map $L_{z}$ to a sublattice of indexण $n=$ $a d-b c$. Each sublattice of index $n$ corresponds to an orbit of $\Gamma(1)$ on

\footnotetext{
${ }^{19}$ If the determinant is not one we change the area of the fundamental domain. See below.

20 This means that there are $n$ fundamental domains of $L_{z}$ in one fundamental domain of the sublattice.
} 
the set

$$
\Gamma_{n}=\left\{\left(\begin{array}{ll}
a & b \\
c & d
\end{array}\right) \mid a, b, c, d \in Z, a d-b c=n\right\}
$$

since an action by $\Gamma(1)$ would not have changed the original lattice $L_{z}$. ( For $n \neq 1, \Gamma_{n}$ is not a group; $\left.\Gamma_{m} \Gamma_{n} \subset \Gamma_{m n}\right)$.

We now define the action of the Hecke operator $T(n)$ on a modular form $f$ as a sum over all the sublattices of index ${ }^{21} n$ :

$$
[T(n) f](z)=n^{\frac{k}{2}-1} \sum_{h \in \Gamma_{n} / \Gamma} f(h(z))\left[\frac{d h(z)}{d z}\right]^{k / 2} .
$$

Using the fact an action by $\Gamma(1)$ merely permutes the terms of this sum ( the left action of $\Gamma(1)$ on the coset $\Gamma_{n} / \Gamma(1)$ ) we can show that $T(n) f$ is also a modular form of weight $k$.

By a right action of $\Gamma(1)$ we can bring any element of $\Gamma_{n}$ to the upper triangular form ; actually we can enumerate the elements of the $\operatorname{coset} \Gamma_{n} / \Gamma(1)$ by $\left(\begin{array}{ll}a & b \\ 0 & d\end{array}\right)$ with $a d=n, b=0,1, \cdots d-1$. For proofs see [18]. A more explicit formula for the Hecke operator is thus,

$$
[T(n) f](z)=\frac{1}{n} \sum_{a d=n} a^{k} \sum_{b=0}^{d-1} f\left(\frac{a z+b}{d}\right) .
$$

In particular, for prime $p$,

$$
[T(p) f](z)=p^{k-1} f(p z)+\frac{1}{p} \sum_{b=0}^{p-1} f\left(\frac{z+b}{p}\right) .
$$

In terms of Fourier coefficients:

$$
f(z)=\sum_{0}^{\infty} f_{m} e^{2 \pi i m z},[T(n) f](z)=\sum_{0}^{\infty} \gamma_{n}(m) e^{2 \pi i m z},
$$

21 We denote $h(z)=\frac{a z+b}{c z+d}$ for $h=\left(\begin{array}{ll}a & b \\ c & d\end{array}\right)$ 
where,

$$
\gamma_{n}(m)=\sum_{d \mid(n, m)} d^{k-1} f_{\frac{m n}{d^{2}}}
$$

In particular"22,

$$
[T(p) f]_{m}=f_{p m}+\delta(p \mid m) p^{k-1} f_{\frac{m}{p}}
$$

It follows then that $T(m n)=T(m) T(n)$ if $m$ and $n$ are coprime. More generally we can show

$$
T(m) T(n)=\sum_{d \mid(m, n)} d^{k-1} T\left(\frac{m n}{d^{2}}\right) .
$$

In particular, the Hecke operators commute with each other. It is also useful that for prime powers we have a recursion relation,

$$
T\left(p^{r+1}\right)=T(p) T\left(p^{r}\right)-p^{k-1} T\left(p^{r-1}\right)
$$

which can be solved in terms of Tchebycheff polynomials $23:$

$$
T\left(p^{r}\right)=p^{\frac{r(k-1)}{2}} U_{r}\left(\frac{1}{2} p^{\frac{k-1}{2}} T(p)\right) .
$$

Thus the $T(p)$ for prime $p$ determine all the $T(n)$.

It is not difficult to establish an inner product on $\mathcal{S}_{k}$ with respect to which $T(n)$ are hermitean:

$$
<f_{1}, f_{2}>=\int_{D} f_{1}^{*}(z) f_{2}(z)[\operatorname{Im} z]^{k-2} d^{2} z
$$

${ }^{22} \delta(p \mid m)=1$ if $p$ divides $m$ and zero otherwise. We use $\sum_{b=0}^{p-1} e^{\frac{2 \pi i m b}{p}}=p \delta(p \mid m)$ to derive this formula.

${ }^{23}$ The Tchebycheff polynomials are defined by

$$
U_{0}(x)=1, U_{1}(x)=2 x, U_{r+1}(x)=2 x U_{r}(x)-U_{r-1}(x) .
$$


where,

$$
D=\left\{z=x+i y \mid-\frac{1}{2} \leq x \leq \frac{1}{2}, x^{2}+y^{2} \geq 1\right\} .
$$

The point here is that $y^{k} f_{1}^{*}(z) f_{2}(z)$ is a modular invariant function, so that it can be integrated after multiplying by the modular invariant volume form $\frac{d x \wedge d y}{y^{2}}$ to get a modular invariant quantity. Of course since each tile contributes the same amount we must restrict the integral to one fundamental domain.

Thus we have a set of commuting hermitean matrices, there is an orthogonal basis of simultaneous eigenvectors, with real eigenvalues: the Hecke forms.

Suppose we have as simultaneous eigenvector a cuspform satisfying,

$$
T(n) f(z)=\lambda_{n} f(z), \quad \forall n
$$

The convention is to normalize an eigenvector by setting the first Fourier coefficient $f_{1}=1$. Taking Fourier coefficients of both sides, we get $f_{n}=\lambda_{n}$ : the Fourier coefficients of a simultaneous eigenvector of the Hecke operators are the eigenvalues. It follows that these coefficients satisfy a multiplicative identity

$$
f_{m} f_{n}=\sum_{d \mid(n, m)} d^{k-1} f_{\frac{m n}{d^{2}}}
$$

There is a Dirichlet series associated to any modular form

$$
f(z)=f_{0}+\sum_{1}^{\infty} f_{n} e^{2 \pi i n z}, \quad \phi(s)=\sum_{1}^{\infty} \frac{f_{n}}{n^{s}}
$$


or,

$$
\phi(s)=\frac{(2 \pi)^{s}}{\Gamma(s)} \int_{0}^{\infty} y^{s-1}\left[f(i y)-f_{0}\right] d y
$$

The modularity of $f$ implies a functional equation ${ }^{4}$ for $\phi$ :

$$
(2 \pi)^{-s} \Gamma(s) \phi(s)=(-1)^{\frac{k}{2}}(2 \pi)^{s-k} \Gamma(k-s) \phi(k-s)
$$

and conversely. The multiplicative property of the coefficients of the Hecke eigenforms yields a product formula:

$$
\phi(s)=\prod_{p} \frac{1}{1-f_{p} p^{-s}+p^{k-1} p^{-2 s}} .
$$

This is reminiscent of the Riemann zeta function $\zeta(s)$. Indeed the theta function, of which $\zeta(s)$ is the Mellin transform, is a modular form of a congruence subgroup of level 2 .

It is of much interest to understand the behavior of the eigenvalues of the Hecke operators. They have been related to the zeros of the zeta function of algebraic varieties over finite fields (Eichler, Sato, Deligne). There are many deep conjectures about the behavior of the eigenvalues $\lambda(n)$ for large $n$. The simplest case is when the dimension of the space of cusp forms is one: when $k=12$ the only cusp form is the function $\Delta(z)$ we introduced earlier. In this case the Hecke operators are $1 \times 1$ matrices: just numbers. From the above it is clear that these numbers

${ }^{24}$ To see this, just take the Mellin transform of the condition for invariance under inversion:

$$
f\left(-\frac{1}{z}\right) z^{k}=f(z) .
$$


are just the Fourier coefficients of the function $\Delta(z)$. In other words, for the case $k=12$, the Hecke operators reduce to the Ramanujan $\tau$-function: $T(n)=\tau(n)$. In fact Hecke discovered these operators by generalizing some ideas of Mordell on the modular form $\Delta(z)$ to the case of higher weight.

From our earlier discussion, we are led to consider the limit of large level where the invariance group becomes abelian. We now present a simple analogue of the Hecke problem for this case of periodic functions.

\subsection{Hecke Operators on Periodic Functions}

Any modular form is periodic so we can expand it in a Fourier series

$f(z)=\sum_{n=1} f_{n} e_{n}(z)$. Thus it is tempting to think of the space of modular forms as a subspace of the space of periodic functions $V$. To make this idea precise, we would like to have a norm on $V$.

The inner product on modular forms given above can be written as

$$
<f, \tilde{f}>=\int_{-\frac{1}{2}}^{\frac{1}{2}} d x \int_{x^{2}+y^{2} \geq 1} d y y^{k-2} f^{*}(z) \tilde{f}(z)=\sum_{m, n=1}^{\infty} f_{n}^{*} \tilde{f}_{m} g_{n m}
$$

where

$$
\begin{aligned}
g_{n m}=<e_{n}, e_{m}>= & \int_{-\frac{1}{2}}^{\frac{1}{2}} d x e^{2 \pi i(m-n) x} \int_{0}^{\infty} y^{k-2} e^{-2 \pi(m+n) y} \\
& \theta\left(y \leq \sqrt{ }\left(1-x^{2}\right)\right) d y
\end{aligned}
$$

is a positive sesquilinear form. We can extend the integral to the fundamental region of the translation group to get an inner product 
on $V$ :

$$
(\psi, \tilde{\psi})=\int_{-\frac{1}{2}}^{\frac{1}{2}} d x \int_{0}^{\infty} y^{k-2} \psi^{*} \tilde{\psi} d y=\sum_{n, m=1}^{\infty} \psi_{n}^{*} \tilde{\psi}_{m} h_{n m} .
$$

In terms of the basis $e_{m}$, we have $h_{n m}$ as a diagonal sequilinear form

$$
h_{n m}=\left(e_{n}, e_{m}\right)=(k-2) ![4 \pi n]^{1-k} \delta_{n, m} .
$$

The Hecke operators are given by quite simple formulae in terms of the Fourier components. We can work out easily their dual action on the basis $e_{m}(z)$ :

$$
T(p) e_{m}(z)=p^{k-1} e_{m p}(z)+\delta(p \mid m) e \frac{m}{p}(z)
$$

for prime $p$. This can then be used extend to them as operators on $V$. The spectral problem for $T(p)$ in the infinite dimensional space $V$ is much simpler than its counterpart in $\mathcal{S}_{k}$. We will solve this simpler problem and see that it has close connections to the theory of random matrices.

How will we recover the Hecke operators on modular forms? We could try thinking of modular forms as a subspace of the space of periodic functions. But under the above inner product on $V$ they will not be square integrable. The reason is precisely modular invariance: each fundamental region contributes an equal amount to the integral, and the region $-\frac{1}{2} \leq x \leq \frac{1}{2}$ contains an infinite number of such regions. Thus, the expansion of a modular form in the basis $e_{m}(z)$ is not convergent in the above norm (., ). On the other hand, the integral for $<.,$.$\rangle corresponding to the sesqilinear form g_{n m}$ also can be extended to an inner product on $V$. It is convergent on modular forms 
but is a degenerate sesquilinear form in $V$ : the integral is restricted

to the region $x^{2}+y^{2} \geq 1$. We can quotient $V$ by the null space of $g_{n m}$ to get a finite dimensional space $\tilde{\mathcal{S}}_{k}$ that is a 'gauge fixed' version of $\mathcal{S}_{k}$. That is, instead of thinking of modular forms as a subspace of $V$, we think of them as a quotient of $V$ by the null space of $g_{n m}$. The 'gauge fixing' amounts to the choice of one particular fundamental region among the infinite number as the domain of the integral. Thus the spectral problem of the Hecke operators on modular forms can be replaced by that on the space $\tilde{\mathcal{S}}_{k}$.

As $k$ grows the dimension of $\mathcal{S}_{k}$ grows linearly with $k$. We will see that in a certain sense the two inner products $g_{n m}$ and $h_{n m}$ approach each other. Thus the discrete eigenvalues of $T(p)$ are so close together as $k \rightarrow \infty$ that they merge to the form the continuous spectrum of $T(p)$ on $V$.

\subsection{Toeplitz Operators}

We now solve the spectral problem for the Hecke operators on periodic functions. It will be more transparent to transform to the orthonormal basis $\mid m>=\left[(4 \pi m)^{1-k}(k-2) !\right]^{-\frac{1}{2}} e_{m}$ we have

$$
T(p) \mid m>=p^{\frac{k-1}{2}}\left[|m p>+\delta(p \mid m)| \frac{m}{p}>\right] .
$$

There is then a simple description in terms of Toeplitz operators. The operators

$$
A^{\dagger}(p)|m>=| p m>, A(p)|m>=\delta(p \mid m)| \frac{m}{p}>
$$


are adjoints of each other and satisfy

$A(p) A^{\dagger}(p)=1, A(p) A\left(p^{\prime}\right)=A(p) A\left(p^{\prime}\right), A(p) A^{\dagger}\left(p^{\prime}\right)=A^{\dagger}\left(p^{\prime}\right) A(p)(128)$

for $p \neq p^{\prime}$. That is, they form a commuting set of Toeplitz operators labelled by the prime numbers. Being isometries $|A|=\left|A^{\dagger}\right|=1$. It follows easily that $T(p)$ are hermitean and that

$$
|T(p)| \leq 2 p^{\frac{k-1}{2}}
$$

The analogue of this inequality on the space of modular invariant functions (rather than periodic functions) is a much deeper statement.

\subsection{Connection to Random Matrices}

The simultaneous eigenfunctions of the $T(p)$ can now be obtained in terms of Tchebychev polynomials. The spectrum is connected with the Wigner distribution for random matrices.

It is enough to study each $T(p)$ separately: the theory 'localizes' completely. To see this, represent each number $m$ in terms of its prime decomposition:

$$
m=\prod_{p} p^{\nu_{p}}
$$

The product is over the set of all primes; $\nu_{p}=0,1, \cdots$ with only a finite number of them being non-zero. Then

$$
\begin{gathered}
A^{\dagger}(p)\left|\nu_{2}, \nu_{3}, \cdots>=\right| \nu_{2}, \cdots \nu_{p}+1, \cdots>, \\
A(p)\left|\nu_{2}, \nu_{3}, \cdots>=\delta\left(\nu_{p} \neq 0\right)\right| \nu_{2}, \cdots \nu_{p}-1, \cdots>.
\end{gathered}
$$


Thus $A(p), A^{\dagger}(p)$ act only on the $p$-th entry.

The Toeplitz algebra is the associative algebra generated by a pair of elements satisfying the relation

$$
A A^{\dagger}=1
$$

The standard representation in terms of an orthonormal basis $\mid \nu>$ $, \nu=0,1 \cdots$ is

$$
A^{\dagger}|\nu>=| \nu+1>, \quad A|\nu>=\delta(\nu \neq 0)| \nu-1>.
$$

This is precisely the representation that we have.

Voiculescu [20] has found a remarkable connection between the theory of random matrices and the Toeplitz algebra. Given any polynomial $f: R \rightarrow R$, define

$$
<f>_{N}=\frac{\int \operatorname{tr} f(X) e^{-\frac{1}{2} \operatorname{tr} X^{2}} d X}{\int e^{-\operatorname{tr} X^{2}} d X}
$$

the integral being over over all hermitean $N \times N$ matrices. Thus $X$ is a hermitean matrix whose matrix elements are independent random variables. Then, Voiculescu shows that

$$
\lim _{N \rightarrow \infty}<f>_{N}=<0\left|f\left(A+A^{\dagger}\right)\right| 0>
$$

There is a probability distribution on $R$, the Wigner semi-circle distribution, such that

$$
\lim _{N \rightarrow \infty}<f>_{N}=\int_{R} f(x) \rho(x) d x .
$$

Explicitly,

$$
\rho(x)=\theta(|x|<2) \frac{1}{2 \pi} \sqrt{ }\left[4-x^{2}\right] .
$$


Thus we see that the Hecke operators $T(p)$ on periodic functions

are hermitean operators whose spectrum is the interval $\left[-2 p^{\frac{k-1}{2}}, 2 p^{\frac{k-2}{2}}\right]$. The (generalized) eigenfunctions are given by Tchebycheff polynomials. The Wigner distribution gives the spectral density. Thus each $T(p)$ behaves like a hermitean random matrix; the different Hecke operators for different prime $p$ commute with each other, so they are not 'free' in the sense of Voiculescu; instead they are statistically independent in the more conventional sense.

\subsection{The limit of Large Weight}

Recall that the main difference between the exactly solvable model above and the the theory of modular forms is that we replaced the sesquilinear form $g_{n m}$ by the simpler one $h_{n m}$. We now show that in the limit of large weight $k$ this is a small correction so that what we obtained above is the asymptotic behavior as $k \rightarrow \infty$.

Note that we can split

$$
h_{n m}=g_{n m}+q_{n m}
$$

where $k$ is the integral over the complimentary region

$$
q_{n m}=\int_{-\frac{1}{2}}^{\frac{1}{2}} d x e^{2 \pi i(m-n) x} \int_{0}^{\sqrt{ }\left(1-x^{2}\right)} y^{k-2} e^{-2 \pi(m+n) y} d y .
$$

If we rewrite this in the orthonormal basis of $h_{n m}$, we will get

$$
\delta_{n m}=\tilde{g}_{n m}+\tilde{q}_{n m}
$$

where $\tilde{q}_{n m}=q_{n m} / \sqrt{ }\left(h_{n n} h_{m m}\right)$ etc. We will show that $\left|\tilde{q}_{n m}\right|$ tends to zero as $k \rightarrow \infty$. 
Now,evaluating the $y$-integral,

$$
\begin{aligned}
\tilde{q}_{n m}= & {\left[\frac{\frac{m+n}{2}}{\sqrt{ }(m n)}\right]^{1-k} } \\
& \int_{-\frac{1}{2}}^{\frac{1}{2}} d x e^{2 \pi i(m-n) x} \frac{1}{(k-2) !} \Gamma\left(k-1,2 \pi(m+n) \sqrt{ }\left(1-x^{2}(1)\right) 2\right)
\end{aligned}
$$

where the incomplete Gamma function is defined by

$$
\Gamma(s, u)=\int_{0}^{u} t^{s-1} e^{-t} d t
$$

We will study this limit as $k \rightarrow \infty$ keeping $m, n$ fixed"2. Now recall that as $s-1>u$, the maximum value of the integrand is attained at its upper limit in this case, so that $\Gamma(s, u)<u^{s} e^{-u}$. Then

$$
\begin{aligned}
\left|\tilde{q}_{n m}\right| \leq & {\left[\frac{\frac{m+n}{2}}{\sqrt{ }(m n)}\right]^{1-k} \int_{-\frac{1}{2}}^{\frac{1}{2}} \frac{1}{(k-2) !} } \\
& {\left[2 \pi(m+n) \sqrt{ }\left(1-x^{2}\right)\right]^{k-1} e^{-2 \pi(m+n) \sqrt{ }\left(1-x^{2}\right)} d x . }
\end{aligned}
$$

Again, replacing the integrand by its largest value ( which is attained at $x=0$ ), we get

$$
\left|\tilde{q}_{n m}\right| \leq \frac{[4 \pi \sqrt{ }(m n)]^{k-1}}{(k-2) !} .
$$

The growth of the factorial beats the exponential growth for fixed $m$ and $n$.

Thus we can see why the distribution of eigenvalues of Hecke operators resemble those of random matrices by our semi-classical approximation method. Moreover we see why the spectrum of $T(p)$ is in the interval $\left[-2 p^{\frac{k-1}{2}}, 2 p^{\frac{k-1}{2}}\right]$.

${ }^{25}$ We should really be estimating the operator norm of $\tilde{q}$. I hope that the arguments here will motivate a more rigorous analysis. 


\subsection{The Poisson Algebra of Modular invariant functions}

We should expect that in the limit of large weight, the theory of modular forms is well-approximated by a classical theory. More precisely the algebra of matrices on $\mathcal{S}_{k}$ should tend to the Poisson algebra of functions on $U / \Gamma(1)$. In particular there will be functions on the upper half plane which are classical approximations to the Hecke operators. These Hecke functions will have vanishing Poisson brackets relative to each other. Their range will give a classical approximation to the Hecke eigenvalue problem. This is another, (manifestly gauge invariant) of studying the limit of large weight.

The upper half plane is a symplectic manifold with symplectic form

$$
\omega=\frac{d x \wedge d y}{y^{2}}
$$

This means that $x$ and $\frac{1}{y}$ are canonical conjugates:

$$
\left\{y^{-1}, x\right\}=1 \text {. }
$$

The set of functions on the upper halfplane form a Poisson algebra with the bracket

$$
\{u, v\}=y^{2}\left(\frac{\partial u}{\partial x} \frac{\partial v}{\partial y}-\frac{\partial u}{\partial y} \frac{\partial v}{\partial x}\right)
$$

The modular transformations

$$
x \mapsto x+1, y \mapsto y
$$


and

$$
x \rightarrow-\frac{x}{x^{2}+y^{2}}, y \mapsto \frac{y}{x^{2}+y^{2}}
$$

are canonical transformations. Thus the space of modular invariant functions is a sub-Poisson algebra. The quotient of this by its center is the algebra of 'gauge invariant observables' if we regard the modular group as a 'gauge group'. We can construct such observables from smooth functions of the upper half plane (vanishing sufficiently fast at infinity) by averaging over orbits. The Maas forms provide nice examples of such 'observables'.

In the limit of large $k$, the Hecke operators should tend to certain modular invariant functions ( which are not holomorphic) that have zero Poisson brackets relative to each other. The range of these functions is the large $k$-limit of the Hecke spectrum. We should also be able to derive a systematic semi-classical expansion in powers of

$\frac{1}{k}$. But this paper is getting long already; I hope to return to these questions in a later publication.

\section{Acknowledgement}

I thank Teoman Turgut and Edwin Langmann warmly for reading through various versions of this paper. Also, Ersan Demilrap brought references [3, 07, 14] to my attention. Thanks are due also to A. Agarwal, L. Akant and G. Krishnaswami for many discussions. The gracious hospitality of the Erwin Schrödinger Institute (Vienna) and the Feza Gürsey Institute (Istanbul), where this paper was written, is also acknowledged.

\section{References}




\section{References}

[1] E. Witten, Nucl. Phys. B160,57 (1979).

[2] E. Brezin and S. Wadia (Ed.), The Large N Expansion in Quantum Field Theory and Statistical Physics: From Spin Systems to 2-Dimensional Gravity, World Scientific (1993).

[3] D.R.Hershbach, Int. J. Quant. Chem. 57, 295, (1996).

[4] S. Guruswamy, S. G. Rajeev and P. Vitale, Nucl. Phys. B 438, 491 (1995) arXiv:hep-th/9406010.

[5] R. J. Henderson and S. G. Rajeev, Int. J. Mod. Phys. A 10, 3765 (1995) arXiv:hep-th/9501080.

[6] S. G. Rajeev, Int. J. Mod. Phys. A 9, 5583 (1994) arXiv:hepth/9401115].

[7] R. G. Parr and W.T. Yao, Density-Functional Theory of Atoms and Molecules, Oxford Science Publications (1989).

[8] S. S. Chern, Complex Manifolds without Potential Theory, Springer-Verlag (1979).

[9] A. Huckleberry and T. Wurzbacher (Ed.), "Infinite-dimensional Kaehler manifolds", Birkhaeuser series on DMV-Seminars, Birkhaeuser, (2001); M. Schlichenmaier, in Moshe Flato Conference Moshe Flato ed. by G. Dito and D. Stenheimer, Kluwer, 2000 math.QA/9910137; and other references given in these books.

[10] J. Mickelsson and S. G. Rajeev, Commun. Math. Phys. 116, 365 (1988).

[11] S. G. Rajeev and O. T. Turgut, Commun. Math. Phys. 192, 493 (1998) . 
[12] A. Pressley and G. Segal, Loop Groups The Clarendon Press, Oxford University, New York (1986).

[13] L.D. Landau and E. M. Lifshitz, Quantum Mechanics Butterworth-Heinemann, (1997).

[14] D. P. Chong ( Ed.), Recent Advances in Density Functional Methods World Scientific, Singapore (1995).

[15] W. Thirring(Ed.), The Stability of Matter: From Atoms to Stars, Selecta of E. Lieb Springer-Verlag (1997).

[16] L. Hormander, Comm. Pure. Appl. Math. 32, 359 (1979); P. Folland, Harmonic Analysis in Phase Space, Princeton U. Press (1989).

[17] L. Akant, G. S. Krishnaswami and S. G. Rajeev, Int. J. Mod. Phys. A 17, 2413 (2002) arXiv:hep-th/0111263; A. Agarwal, L. Akant, G. S. Krishnaswami and S. G. Rajeev, arXiv:hepth/0207200, to appear in Int. J. Mod. Phys.

[18] T. Apostol, Modular Functions and Dirichlet Series in Number Theory, Springer-Verlag (1990).

[19] P. Sarnak, Some Applications of Modular Forms, Cambridge University Press, (1990); P. Sarnak and N.M. Katz, Random Matrices, Frobenius Eigenvalues and Monodromy, American Mathematical Society, (1999).

[20] M. L. Mehta, Random Matrices Academic Press, New York (1967); D. Voiculescu, K. Dykema and A. Nica, Free Random Variables American Mathematical Society, Providence Rhode Island (1992). 